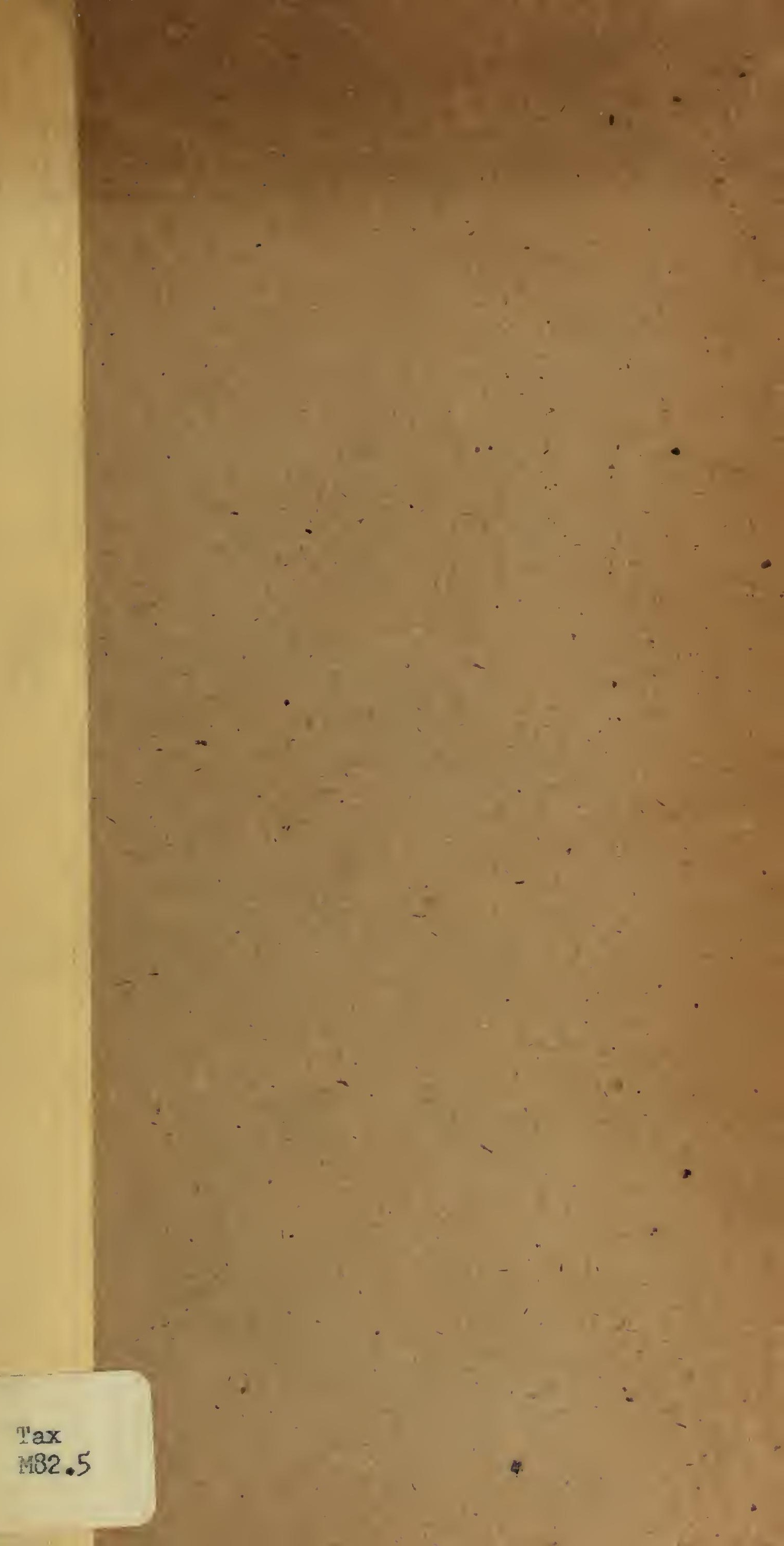



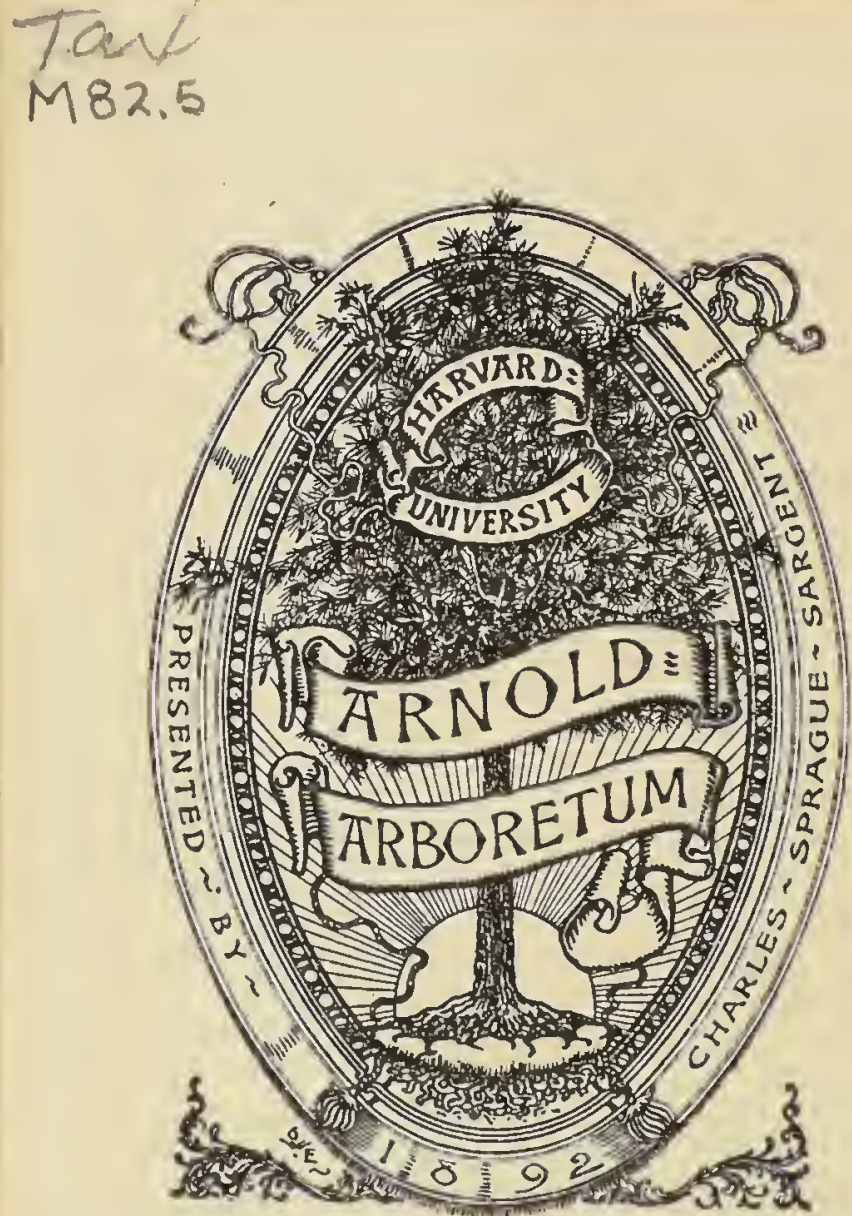


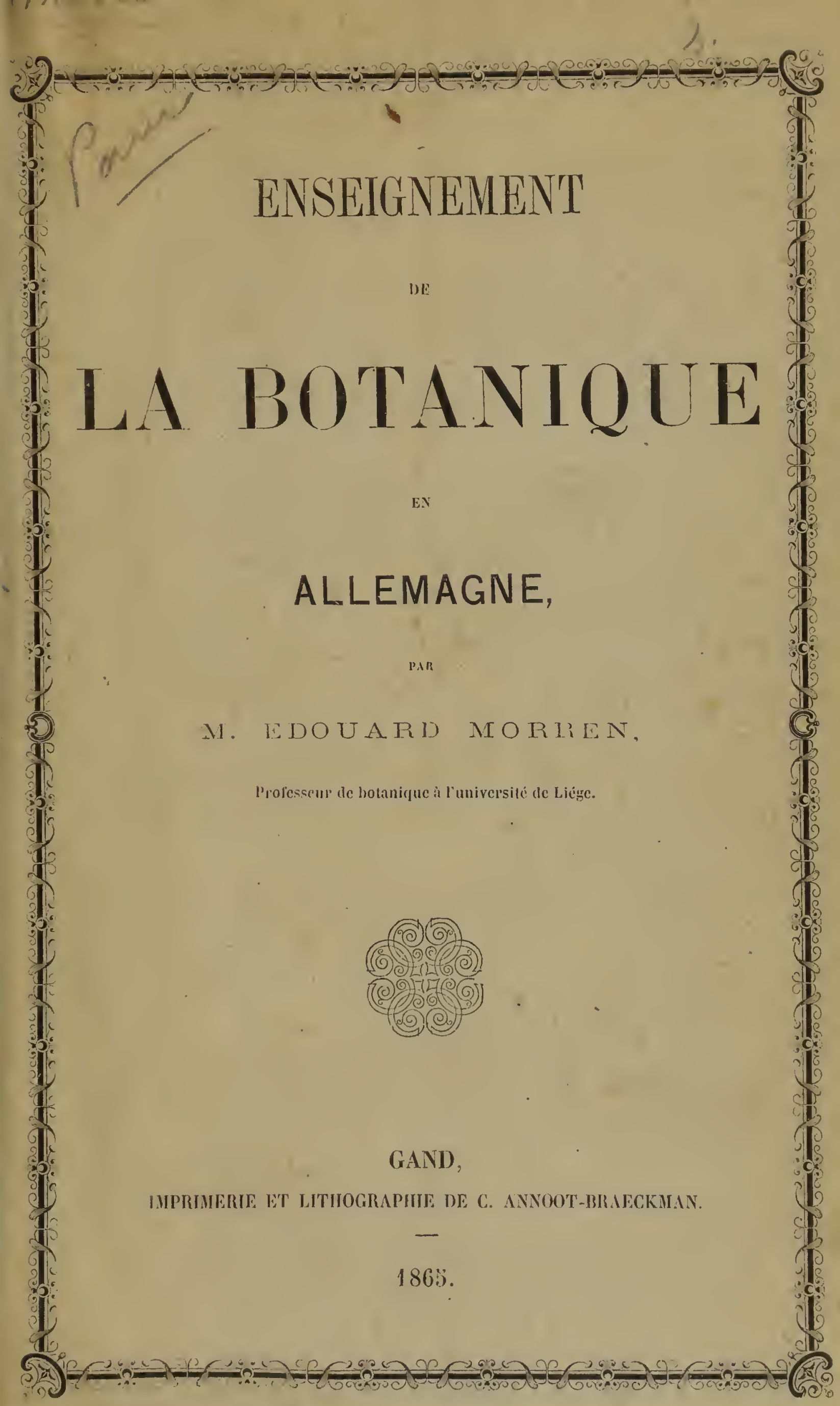





\section{ENSEIGNEMENT DE LA BOTANIQUE}

\section{EN ALLemAGNe.}





\title{
ENSEIGNEMENT
}

DE

LA BOTANIQUE

EN

\section{ALLEMAGNE,}

\author{
p..n \\ M. LDOUA B. NOHLEN. \\ rrofessent de butanique à l'universilé de Liége.
}

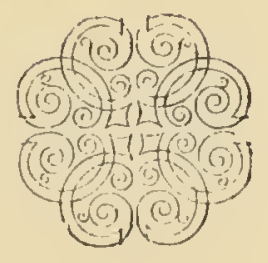

(i.) (1)

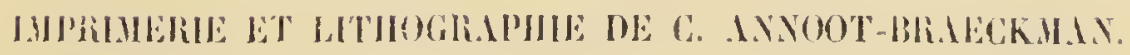

$186 \%$ 
27292 


\section{PREFACE.}

Ces quelques pages eoncernent une question qui fait l'objet de nos constantes préoceupations. Nous les avons écrites, au courant de la plume, en nous abandonnant aux sentiments de notre conviction. Nous y exprimons nos pensées avec franchise et, nous a-t-on dit, avee une certaine énergie. Cependant, si notre plume nous a bien servi, personne ne pourra se méprendre sur nos intentions. Nous espérons trouver chez tous nos collègues autant de bienveillanee que nous éprouvons de reconnaissance pour nos anciens professeurs.

Nous avons livré notre écrit à l'impression afin de pouvoir le eommuniquer à des amis et à des confrères qui s’intéressent ainsi que nous, à l'avenir des seiences en Belgique et à la prospérité des Universilés, et aussi afin de stimuler l'ardeur' et la confiance des jeunes savants.

Enouarn Morren. 

Toutes les personnes qui ont étudié la question de l'enseignement des scienees en Belgique, question maintes fois poséc par le gouvernement et qui n'a jamais été résolue par les clambres, s'accordent toutes à reconnaitre que des améliorations sont néeessaires. On pourrait croire quelquefois que l'esprit scientifique n'anime pas assez notre jeunesse stndicuse. Sans méconnaitre que les tendances professionnelles et utilitaires exercent ehez nous comme partout leur influence, nous croyons plulôt que ces aspirations sont étouffées faule de pouroir se produire. II n'y a pas assez de positious pour les jeunes savants; il n'y a pas de stage pour le professorat, nulle transition ne relie l'étudiant, le docteur même, au professeur; les jeunes gens de talent qui, entrainés par leur amour de la seience, veulent consacrer leur vic aux études, ne trouvent pas de positions en rapport avee leur jeuncsse et leurs aspirations. Aussi l'enseignement supérieur se recrute-t-il péniblement sans sortir de notre nationalité.

Il serait facile de repousser les reproches qu'on s'est laissé aller à adresser aux Belges et de montrer que pour porter remède au mal il suffirait au gouveruement d'augmenter un peu le nombre des eliaires scientifiques dans ses universités et surtout de eréer, en faveur des jeunes docteurs, quelques positions secondaires autour des professeurs en titre.

Ou proclame ici comme en France et ailleurs, la valeur seientifique de l'Allemagne. En effet, le nombre des savants y est considérable; l'esprit seientifique y est ardent, vivace; les universités sont bien loin, en général, d'être embarrassées pour recruter les rangs de leur personnel enseignant. On nous eile, avee raison, leur organi. sation, comme un modèle à suivre chez nous. Nous ne viendrons pas redire après les études approfondies de MM. Loomans et Banningr dont nous partageons les opinions générales(1), quelles sont l'origine

(1) Rapport sur l'enseignement supériem en Pinsse, par M. CH. Loonus. - Auniles les Univers. de Belgique, 2e série, t. I. 1861.

Rapport sur l'organisation et l'enscignement de l'Université de Benlin, far ill. Eu.

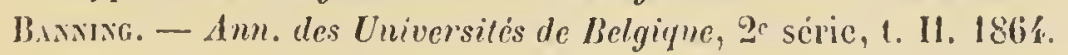


et l'organisation des universitcs allemandes, nous invoquons toutefois ces rapports lumineux comme l'introduction néeessaire pour la lecture de notre écrit.

Nous sommes heureux de reconnaître que le gouvernement, justement préoccupé de celte importante question, a beaucoup fait pour encourager l'espril scientifique. Les tentatives et les essais pour pourvoir au recrutement du corps professoral supéricur n'ont pas manqué. Institution des agrégés, création du doctorat spécial et en ec moment mème des chaires universitaires ouvertes à tous les docteurs qui voudront y donner un cours nouveau. Nous craignons fort que cette dernière mesure si libérale ne reste aussi stérile que les précédentes pour des raisons qu'il est inutile d'indiquer ici. En tenant compte de l'état des choses tel qu'il existe et de notre esprit national, nous croyons qu'il faudrait chercher à grouper autour des universités un grand nombre de jeunes savants en e réant pour eux des emplois de préparatenr's, conservateurs, répétiteurs etc., et en leur permettant, sans prejudice des autres et sous certaines conditions, de prendre part à l'enseiguement.

Nous craignons de nous laisser entrainer trop loin. Notre but en prenant la plume a été simplement de faire connaitre, par quelques exemples, et par des faits que nous avons été recueillir sur les lieux, l'étal actuel de l'enseignement de la botanique en Allemagne.

Il y a en Prusse 7 universités, à Berlin, Bonn, Breslau, Greiswald, IIalle, Konigsberg et Munster; en Autriche 4, savoir a Gratz, Inspruck, Prague, Vienne; J en Bavière, à Erlangen, Munich et Wurzbourg; 1 en IIanorre, à Goettingue; 1 en Saxe, à Leipzig; 1 en Wurtemberg, à Tubingue; 2 dans le grand-duché de Baden, à Fribourg et à IIeidelbergy; 1 dans le grand-duclı́ de Hesse-Darmstadt, à Giessen; 1 dans le grand-duché de Ilecklembouror-Sehwerin, ì Rostock; 1 dans le grandrluché de Saxe-Weimar-Eisenach, à Iéna; 1 dans l'électorat de Ilesse, a Marbourg; 1 daus le IIolstein, à Kiel. Soit 24 universités en Allemagne.

La Suisse en compte 5, à Bâle, Berne et Zurich.

La IIollande 5 , ̀̀ Groningue, à Leyde, à Utrecht.

La Belgique 4, à Bruxelles, Gand, Liége et Louvain (1).

$\mathrm{Or}_{1}$, tandis que dans chacune de nos quatre universilés de Belgique il n'existe qu'un seul professeur de botanique, lequel est obligé de renferuer tout son enseignement dans un seul cours, chaque université allemande possède au moins deux, en général trois et souvent un plus grand nombre de bolanistes. Chacuu professe plusicurs cours, de sortc que ceux-ci s'élèvent parfois jusqu'à 13 ou 20.

(1) Remar'fuons qu'il existe en Allemagne, comme dans la plupart des États, des jarlins botaniques importants en dehor's des universités, par exemple ì Carlsruhe, à Mresite, al Itanorre, à Stultgardt, cle. 
Les diverses branches de la science si vaste de l'étude des végétaux, oceupent dans les programmes allemands la place qui leur est due. Nous citerons par exemple : la bolanique médicale, la cryptogamic, la géograplice des plantes, l'anatomie végétale, etc.

La situation faite chez nous à la bolanique n'est pas en rapport avec son importance. Tandis que les deux autres règnes de la nature, le règne inorganique et le règne animal, occupent un grand nombre de clıaires, il n'en est donné qu'une seule pour le règne régétal tout entier, it tous ses degrés el sous tous ses aspects.

En Allemagne il exisle à peu près pour chaque branche et en parliculier pour la bolanique des professeurs ordinaires, des professeurs extraordinaires et des agrégés. Chacun ouvre plusieurs cours et il jouit quant à leur choix d'une gerande liberté. De là une véritable émulation. Le principe de la coneurrence est largement admis. Le même cours est parfois donné deux fois et même trois fois et à la même lıcure. Chaque professeur titulaire doit, outre son cours proprement dit, en donner all moins un autre. Il choisit pour cela des sujets de prédilection, qu'il a particulièrement approfondi et trouve ainsi le moyen de faire de véritables disciples. 11 ourre done des cours privés et même des cours particuliers pour lesquels on prend des inscriptions isolées. Le cours est-il bon, les auditeurs s'empressent et le professeur est sous tous les rapports rémunéré de ses peines. Il varie d'ailleurs ces cours suirant qu'il étend lui-même ses éludes.

Les élèves de leur côté jouissent de la même liberté : ils peuvent s'inscrire à tel ou tel cours à leur gréé, et il leur suffit, lorsqu'ils se présentent à l'examen, de justifier leur assiduité à l'un ou l'autre cours des branches qu'ils ont dû étudier.

Nous laisserons parler les faits : c'est notre seul but aujourd'hui, (:lacun pouvant tirer les conséquences naturelles qui découlent de la connaissance des programmes allemands, en ee qui concerne l'enseignement de la botanique.

\section{UNIVERSITÉ DE BERLIN.}

A l'université de Berlin, pendant le semestre d'hiver $1864-186 \ddot{3}$, le: Dr A. Braun, professeur ordinaire :

Dirigera un cours public de conversations botaniques le vendredi dr 6 a 7 heures;

Donnera un cours privé de botanique spéciale, cxposant l'ensemble des familles du règne végétal et particulièrement les plantes qui sont en usage en médecine ou dans l'industrie: le mardi, le mercredi, le jeudi et le vendredi de 5 à \& heures.

Se propose de donner des conférences particulières sur l'observation et la détermination des plantes cryptogames. 
Le Dr O. Benc, professeur extraordinaire:

Fera des démonstrations publiques pour l'observation au microseope des médicaments régćtaux.

Donnera deux cours privés:

L'un de botanique spéciale, traitant des familles et spécialement des plantes officinales et utiles, at pour lequel il fera usage de son herbier, l'autre de pharmacognosic. Le premicr comportera six, le sccond quatre lecons par sentaine.

Le Dr II. Karsten, professem extraordinaire :

Donnera une conference botanique le lundi de chaque semaine de 6 à 7 heures.

Et deux cours privés, saroir :

La botanique médicale et l'examen spécial des organes de certaines plantes qui sont employées en pharmacic (six fois par semaine).

Lá pliarmacognosie; \& lecons par semaine.

Le $\mathrm{D}^{\mathrm{r}} \mathrm{C}$. Kocu, professcur extraordinaire :

Deux cours publics :

$1^{\circ}$ Les familles naturelles, une fois la semaine.

2 Des conférences à l'institut agronomique.

Un cours privé :

Botanique économique el maladie des plantes, quatre fois par semaine.

Nous trourous aussi parmi les cours des professeurs extraordinaires, celui du Dr II. Schaur, sur les insectes utiles ou nuisibles en agriculture.

Puis les cours des agrégés ou Privatim docentes, lesquels, quant à l'enseignement, sont sur le même pied que les professeurs en titre.

Le $\mathrm{D}^{\mathrm{r}} \mathrm{J}$. IIsNstein donne quatre fois par semaine des lecons d'anatomic et de physiologie végétales, pendant lesquelles il fait usage du microscope.

De plus un cours particulier de botanique pratique, surtout d'exercices au microscope. Ce cours se tient à l'Herbier royal tous les jours de 11 heures à 2.

Le $\mathrm{D}^{\mathrm{r}} \mathrm{A}$. Thuen enseigne les principes de l'Economic rurale et fait arec ses élèves des excursions dans les environs de Berlin.

Quelle multiplicilé de moyens d'instruction! Chaque professeur donne plusicurs cours en choisissant les sujels qu'il a le micux étudiés. Il y a d'ailleurs pour lui obligation de professer outre un cours public, un autre cours privé. Les élèves peurent choisir leurs matières et trouront le moyen d'approfondir ce dont ils veulent se faire une spécialité. Il y a émulation, concurrence, même entre les professeurs et tout le corps enseignant à ses dirers degrés. Nous trouvons le même cours donné par plusicurs sarants, mais chacun se placant à un point de vue spécial, il peut y aroir intérêt ì les écouter lous deux. Les cours privés sont rétrilués par les auditeurs. Présentent-ils de l'intérêt et sont-ils bien donnés, 
les élèves vienuent s'injerire, payent 2 ou 5 Fredéries d'or et le professeur est rétribué pour son travail. Il y a, en outre, des exereices praliques avec les herbiers ou le mieroscope et pendant lesquels les élèves se trouvent dans la familiarité de leur professeur et de la seicnee.

Nous n'avons parlé que du semestre d'hiver actucl. Dans le semestre d'été qui vient de finir (1865 à 1864), nous trouvons encore un grand nombre d'autres eour's.

Le professeur Schultz-Schultzenstein a donné deux cours de bolanique llićorique el pratique dans ses rapports aree la physiologie.

Botanique générale (morphologie), anatomie et physiologie des plantes, par le prof. Braun, six fois la semaine.

Des démonstrations pratiques au jardin botanique tous les samedis soirs et des herborisations aux environs de Berlin.

Botanique générale et systématique à l'aide de démonstrations sur les plantes vivantes par le prof. Berg (six fois la scmaine, eours privé).

Le même conduit aussi des herborisations.

Le prof. Kansten donne un cours exactement sous le même litre que le précérlent.

Le Dr Hansteis professe spéeialement sur les plantes médieinales ou éeonomiques, dont il montre des exemplaires vivants, et il fait des herborisations.

Le prof. Braun domne encore le jeudi de $̋$ à 6 lieures un eours parliculier sur la méthode naturelle de elassification végétale.

Le Dr Pringsieim, aujourd'hui á Iéna, domnait un eours particulier de reeherches sur l'anatomic et la pliysiologie végétales.

Le Dr Hanstein un autre de recherehes mieroscopiques.

Le prof. Koch donnait un cours de botanique agricole et sur l'anćlioration du sol et dirigeait aussi des excursions seientifiques.

Nous eitons les programmes des autres universités sans aueun commentaire.

Il y a aussi en Allemagne des universités faibles, où l'enscignement de la botanique cst tombé entre les mains d'un scul professeur : nrais ce sont les moins importantes, ou bien ectte situation est toute passagère. Les facultés out le droit de sigualer au gouvernement les sciences dont l'enseigncment devrait être renforé chez elles. Il est rare que celui-ei I'y portc pas remède.

Parmi les cours portés au programme des universités allemandes, il en est, nous sommes les premicrs à le constater, qui unéritent à peine ce nom et qui, en réalité, peuvent rentrer daus le cours de botanique générale. Iais il en est d'autres qui doivent être professés séparément ct dont le inanque est à déplorer cher nous. 


\section{UNIVERSITE DE BONN.}

\section{semestréc d'été de 1863.}

1. Botanique géuérale, par le prof Treviranus.

2. Bolanique générale et systématique, par le prof. Scisscirs.

3. Éléments de botanique systématique, anatomique et physiologique, par te Dr Ihlomemand.

4. Système naturel des végétaux indigènes, par le prof. Treveranus.

3. La féeondation des plantes : prof. Schacht.

6. Exeursions botani(ques, par le mime.

7. IIerborisations el démonstrations, par le prof. IliLdebrand.

\section{Semestre d'hiver 1863 à 1864.}

8. Botanique cryplogamique, prof. Treviranus.

9. Sur les cryptogames, Dr II hedranu.

10. Plantes parasites, prof. Schachit.

11. Les plantes offieinales, par le même.

12. Pharmaeognosie botanique, par le mêne.

15. Anatomic et physiologie végétales, sous forme de démonstrations au microseope, Dr IILLEBRAND.

\section{UNIVERSITÉ DE BRESLAU.}

\section{Semestre d'hiver 1864.}

DP II. R. Goeppert, prof. ordinaire.

1. Exereiees de botanique deseriptive et de recherches mieroseopiques. Cours particulier, plusieurs fois par semaine.

2. Botanique eryptogamique et démonstrations mieroseopiques, 2 fois par semaine. Cours publie.

3. La flore primitive et spécialement des plantes fossiles propres à chaque formation, une leçon par semaine. Cours publie.

4. Les plantes exotiques dı jardin botanique. Cours publie consistant en conférenees pratiques dans les serres.

5. Anatomie, morphologie et physiologie végétal es, avee démonstrations mieroseopiques et expérienees, 5 leçons par semaine. Cour's privé.

6. Flore phanérogame d'Allemagne et sa distribution géographique, 2 lccons pa' semaine. Cours privé.

1) Ferd. J. Coirs, professeur extraordinaire:

1. Reproduction des plantes, une leẹon par semainc. Cours public.

2. Prineipes de botanique agricole, 5 leeons par semaine. Cour's privé.

3. Anatomic et biologic des plantes, 5 lecons par semaine, plus des excrcices au microseope. Cours privé. 


\section{Semestre d'été 1864.}

Dr H. R. GOEPERT :

1. Excreiees de micrographie pharmacologique, unc lcẹon par semainc. Cour's public.

2. Des plantes oflicinalcs et de leurs propriétés, 4 lecons par scmaiuc au jardin bolanique. Cours privé.

5. Exeursions botaniques dans les cnvirons de Brcslau, une fois par semainc. Publiques.

4. Botanique géıérale, 4 leẹons par semaine au jardin botanique. Cours privé.

5. Botanique spéeiale et exposition des familles naturelles, lecons par scmaine. Cours privé.

D) Ferd. Coun :

1. IIstoire des Algucs, deux fois par semainc. Cours partieulier.

2. Géographic des plantes, deux fois par semaine. Cours public.

3. Élémonts de botaniquc générale, \& leẹons par semainc. Cours privé.

4. Familles naturelles et lierborisations, 3 fois par semainc. Cours privé.

1) G. G. Koerbeil :

1. Classification des Lichens, 2 leçons par scmaine. Cours particulier.

\section{UNIVERSITÉ D'ERLANGEY.}

\section{Semestrec d'été 1863.}

Prof. extr. De Scanizlein :

1. Botanique médicale, 5 leẹons par semaine.

2. Botanique eryptogamique : cours privé, 2 lecons par semaine.

3. Exercices pratiques pour la détermination ct la description des plautes, 2 leçons par scmaine.

4. Ilerborisations dans les cnvirons d'Erlangen, une fois par semaine.

\section{Semestre d'hiver 186 f.}

Prof. Schnizheix :

8. Organographie, anatomie ct physiologic des plantes, 6 lcçons par scmaine.

(i. Microscopic des substances végćtales cmployécs en pharmacic. Cours privé.

\section{UNIVERSITE DE FRIBOURG (EN BRISGAU).}

\section{Semestre d'été 1863.}

Botanique mćdicinale, 5 lcçons par semainc. Professcur ordinaire be Banr.

Botanique spéciale, 5 leçons par scmainc. Le même.

Exercices pour l'élude des plantes. Cours particulier. 


\section{Semestre d'hiver 1863-1864.}

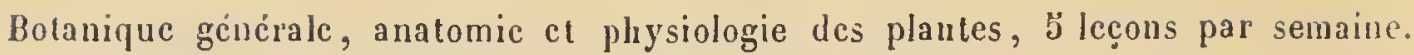
Prof. De Bany.

Sur les plantes cryptogames, \& leçons par semaine. Icc mêmc.

\section{UNIVERSITÉ DE GIESSEN.}

\section{Semestre d'hiver 1862 à 1863.}

Botanique générale, anatonie et physiologic des plantes, 4 leçons par scmainc et démonstrations au microscope. Prof. ordin. Dr Ilofrasix.

Ilistologie, morphologie et physiologie des plantes, 4 leçons par scmainc. Prof. extr. D. Rossmans.

Pratique de la botanique, une Icegon par scmainc. Prof. IIorfasix.

Conversations botaniques, une leẹon per scmainc. Le même.

Botanique pharmaceutique, 4 leçons par semaine. Le mème.

Bolanique forestière, 4 lecons par semaine. Prof. Rossmaxn.

Structure des bois, 2 hcures par scmainc. Lc mêmc.

Répétitions et interrogations de botanique, par le mème.

\section{Semestre d'été 1863.}

Morphologie, tcrminologic botanique et familles naturelles, détermination des plantes et herborisations, $\overline{3}$ leçons par semainc. Prof. Hoffuaxs.

Bolanique pharmaceutique, avec excursion, etc., par le même.

Botanique spécialc, particulièrement des plantes pharmaceutiques, avec herborisations, $\ddot{b}$ lecons par semainc. Prof. Rossmaxx.

Bolaniquc forestière, avcc exeursion, par le même.

Bolanique cryptogamique, par le même.

\section{UNIVERSITÉ DE GOETTINGUE.}

\section{Semestre d'été $186 \AA$}

\section{Cours ordinaires.}

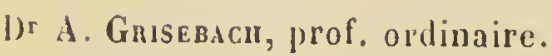

1. Botanique générale et spéciale, avec herborisations, six lecons par semainc.

2. Botanique médieale, quatre leçons par scinaine.

5. Exercices pratiques de botanique descriptive. 
I)r F. Th. Bantung :

1. Botanique spéciale, précédée des éléments de lotanique générale; 6 leçons par semaine.

2. Botanique médicale, 5 leçolis par semaine.

3. Botanique industrielle, 4 leegons par semaine.

4. Exeursions botaniques.

3. Démonstrations au jardin botarique.

Cours des agrégés.

Dr S. Lantzius-Benivga.

1. Botanique générale et spéciale, 6 leçons par semaine, avec interrogations, démonstrations et herborisations.

\section{Semestre d'hiven 1865.}

\section{Cours ordinaires.}

Dr A. Grisebicil :

1. Anatomie et physiologie des plantes, en mème temps que physiologie agro. nomique, leçons par semaine.

2. Histoire universelle de la nature, leçons par semaine.

3. Ilistologic botanique, enseiguée par des démonstrations pratiques. Cours privé.

D) Bartling :

1. Démonstration des plantes eultivées dans les serres du jardin botanirque, une fois par semaine.

2. Histoire des végétaux eryptogames, \& leçons par semaine.

3. Exeursions botaniques.

\section{Cours des agrégés.}

i)r S. Lantzius-Beninga :

1. Comparaison des végétaux phanérogames et eryptogames, 2 leẹons par semaine.

2. Physiologie el anatomie végétales, avee démonstrations au mieroseope, \& leẹons: par semaine.

5. Répétitions de botanirgue générale et spéciale.

\section{UNIVERSITÉ D'HEIDELBERG.}

\section{Semestre d'hiven 1863 ì 64.}

Morphologie et physiologie des plantes, \& lecons par semaine. Prol. IlofaeIsten.

Sur les eryptogames, 2 leẹous par semaine. Par le mème.

Excreices pratiques de phytotomie el d'expérimentation, 2 leçons par semaine. Le même.

Botanique spéciale, 2 leçons par semaine. Prof. Scumidt.

Répétitions el interrogations de botanique, par le Dr Autes. 


\section{Senestre d'été 1864.}

Botanique générale el spćciale, ö lecons par semainc. Prof. Hofaelster.

Botanique générale ct herborisation. Dr A HLEs.

Physiologie cxpérimentale des vénćtaux, I leçon par semaine. Prof. Hofmeister.

Exercices pratiques de plyytotomic et de micrographie, 2 Iccons par scmaine. Par le mème.

Bolanique médicinale, 5 lecons par semaine. Dr Aubes.

Exercices pratiques pour la dénomiuation des plantes, „2 leçons par semaine Le même.

\section{UNIVERSITÉ DE IENA.}

\section{Semestre d'été 1863.}

H. J. SCHLEuEX, prof. ordinaire:

Bolanique, älecons par semaine. Cours prive.

Cir. Ed. Laxgethal, prof. ordinaire:

Botanique économique et physiologic des plantes, 6 leçons par semaine. Cours particulier.

Les champs el les prés, éludes agronomiques avce excursions.

J) En. IlaLlier, agrégé :

Botanique mćdicalc el pharmaccutique, ’̆ leçons par semainc. Cours priré.

Exercices phytotomiques. Cours particulicr.

Ifrrorisations aux euvirons de léna.

\section{Semestre d'hiven 1861.}

U) E.s. HAt.lien :

Botanique pharmacognostique, \& leçons par semaine.

Réfuétitions et interrogations, par lc même.

L'enseignement de la hotanique se trouvait en 1865-1864 en souffrance ì l'université de Iéna, par le départ du prof. Sehleiden pour lorpat et la nomination du prof. Pringsheim.

\section{ITIVERSITE DE KONIGSBERG.}

\section{Semestre d'été 1863.}

l'ar le lis Cismar, prof, ord. :

1. Phytotomic pratique ct recherches microscopiques, 1 lecon par semaine.

2. Botanique générale ou élémcnts de morphologic, anatomie, physiologic, tcxo logic, éclaircics par des olservations au microscope, des démonstrations sur les plantes rivantes el des exculsions hotaniques. 6 lccons par semaine. Cours privé.

3. Plantes officinales, $\overline{7}$ leçons par semainc. Cours privé. 
Par le Dr Samo :

1. Excursons hotaniques, une lois par semaine.

2. Botanique spéciale, $̋$ leẹons par semaine. Cours privé.

\section{Semestuoc d'hiven 1864.}

Par le Dr Caspani:

1. Physiologie des plantes, \& lecons par semaine. Cours pricé.

2. Pharmacognosic, 5 le egons par semainc. Cour's privé.

3. Répétitions d'anatomie végétale.

Par le Dr S.riso :

1. Botanique générale, \& leẹons par semaine. Cours privé.

2 Sur les eryptogames.

Par le Dr Scmuent .

1. Théoric des cultures, flecons par semaine. Cours privé.

\section{UNIVERSITÉ J) LEIPZIG.}

\section{Semestre d'été 1861 .}

Lxereices pratiques de recherches botaniques, 2 leçons par semaine, par le professeur ordinaire Dr Megrexius. Ilerborisations.

\section{Semestre d'hiver 1865.}

Anatomie et physiologie des plantes, par le mème, 4 leegons par semaine. Sur les vćgétaux cryptogames, par le même.

\section{UNIVERSITE DE MARBOURG.}

\section{Semestre d'été 1863.}

P'rofesseur IVIGaxd.

Botanique générale et démonstrations au mieroscope, 5 fois par scminine.

Botanirque populaire, 1 fois par semaine.

Les plantes officinales et utiles, 2 leeons par semaine.

Exereices botaniques, 2 fois par semaine. Cours privé.

Excursions hotaniques, 1 fois par semaine. 


\section{Semestre d'hiven 1863-61.}

Géographic el palicontologic végétales, \& lecons par semaine. Les familles des cryplogames, 5 lecons par semaine. Pliarmacognosic, 2 lecens par semaine.

Exerciees pharmacognostiques, 1 fois par semaine.

\section{UNIVERSITÉ DE ROSTOC.}

\section{Semestre d'hiver 1862-63.}

Dr. J. ROEPER:

1. Plantes cryplogames, 2 lecons par semaine. Cours public.

2. Familles du liegne végétal, 2 lecons par semaine.

Dr E. BECKER :

1. De la enlture des forêts.

\section{Scmestre d'été 1863.}

Ur lioeper :

1. Elćments d'anatomic végélale; 1 leçon par semaine.

2. Botanique générale et exercices pratiques, ( lecons par semainc.

5. Des plantes sauvages dans les champs el les bois, 2 lecons par sumaine.

1. Excursions botaniques.

Dr BeGKER :

1. Culture des plantes agrieoles, 4 lecons par semaine.

\section{UNIVERSITÉ DE TUBINGUE.}

\section{Semestre d'éte 1862.}

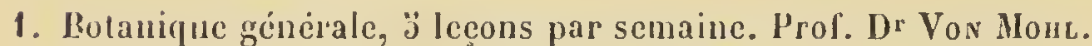

2. Flore de IVurtemberg, avec démonshations sur les plantes vivantes ou séches, 乌lecons par semaine. Prof. Dr Sigwart.

3. Exercice pour l'élude des planles, 2 ou 3 fois par semaine. Prof. Dr Vox Molic.

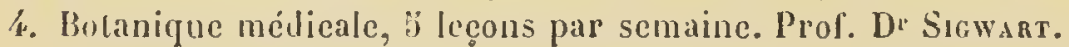

5. Botanique médicinale et pharmaceutique avee démonstrations, 3 lecous par semaine. Prof. Dr llenkel.

\section{Semestre d'hiver 186 s.}

1. Chimic agricole, ö fois par semaine. Prof. Sigwant.

2. Chimic pharmacentique, 5 fois par semaine. Prof. Hexker.

7. Analomic el physiologic des végélanx, : Iregns par semainc. Prof. H. Vox Mlont.

\& Sur les cryploganes, ¿2 ou 3 lois par semainc. Par le mème. 


\section{UNIVERSITE DE VIENNE.}

\section{Semestie d'été 1864 .}

Morphologie el classification des plantes phanérogames; 3 fois la scmaine, par le prof. Dr Fin. Fexzl.

Exereices pratiques pour la détermination et la deseription des plantes, 5 fois la semaine, par l'agréré Jos. Böıs.

Repetitorium de botanique, 5 fois la semaine, par l'agrégé Dr G. Reicuandr.

Sur les plautes médiciuales, 2 fois par semaine, par le mème.

Exereiecs pour la deseription et la détermination des plantes indigènes et herborisation dans les environs de Vienne, 2 fois la semaine, par le même.

Eeonomic rurale, plantes agricoles, ete., 5 fois la semaine, par le prof. An. Firens.

\section{Semestre d'hiver 864 à 1865.}

Analonic et physiolngie des plantes, démonstrations au microscope, 5 fois la semaine, par le prof. Jos. Uager.

Démonstrations sur l'analomie et la plysiologie des plantes, une fois la semaine, par l'agrégé Dr Jos. Böus.

La morphologie et la classifieation des champignons el spécialement de la distinetion des espèces utiles et nuisibles, 2 fois la semaine par l'aggrégé Reicuand( 1 ).

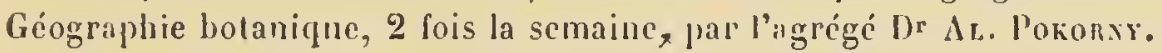

Agronomie, 4 fois la semaine, par le prof. Dr An. Fuchls.

\section{UNIVERSITÉ DE WURZBURG.}

\section{Semestre l'hiver 1861-62.}

Dr LAEIBLEIN :

1. Bolnnique générale, oryanographie et physiologie des plantes.

Dr SCHExk :

1. Ilislologic végétale. Cours partieulier.

2. Histologic et physiologie des plantes.

5. Sur les plantes fossiles.

\section{Semestre d'été 186\%.}

D) LEHLEIN :

1. Répélitions de bolaniqua générale.

Dr Scuriti :

1. Botanique générale, 6 ieçons par scmainc.

2. Botanique médiciuale, li lecons par semaine.

5. Botanique spécialp, 3 lecons par semaine.

(1) L'année précédente M. Reichardt avail donuć un eotres special sul les Mousses al les Fongeres. 
Que l'on se reporte maintenant en Belgipue el que l'on compare ce qui cxiste en Allemagne avec les programmes de nos universités!

El l’on s'élonic de ne pas roir plus souvent surgir de véritables savants, de ne pas avoir des éludes plus solides : el l'on voit nos jeunes botanistes ĉlre obligés d'aller eliercher à l'élranger le complément indispensable de leurs éludes :

Mais ce qui nous étonne, nous, e'est de roir eneore une pléiade de jeunes savants se former en Belgirque.

Nous ne faisons pas ici le procès à la botanique seulement. Nous la prenons comme cxemple, mais ce que nous disons de celte science est également rai de loutes les autres, des sciences naturelles en parliculier.

L'enseignement devient chez nous trop professionnel. Dans notre organisation sociale, l'institution de nos universités, gouvernementales ou libres, scmble avoir surlout pour but de pourvoir la Société d'avocats, de médecins, d'ingéniemrs. Sous ec rapport clles satisfont pleinement ì ce qu'on attend d'elles et, en réalité, elles dotent notre pays d'un corps médical, d'un barreau, d'une magistrature el d'autres professions libérales qui ne le cèdent à ceux d'aucun autre pays. Mais ee ne devrait pas être le scul but des unirersités. Les facultés de philosophic et des sciences sont trop sacrifiées respectivement à celles de droit et de médecine auxquelles elles conduisent.

Dans la plupart des cours on a énorménent à enseigner el l'on s'arlresse à un public nombreux et hétérogène. Ce que les cours gagnent ainsi en ćtendue il le perdent en profondeur. Ils suffisent amplement pour la préparation aux carricres professionnelles, mais nous les croyons sourent insuffisants pour les aspirations scientifiques.

Les universités doivent avoir un autre but el pourvoir à un second hesoin de la société, celui de fournir au pays des sarants, comme les académies de peinture el de musique doivent faire éclore des peintres, des sculpteurs et des eompositeurs. Or c'est précisément ce but là qu'elles ont peine à atteindre pour ne pas dire plus. Lá nous semble toute la question de l'enscignement supéricur el s'il était porté remède à ce mal là, nous n'aurions plus rien à désirer et bientôt plus rien à énvier aux nations les plıs farorisécs. Lc prétendu abaissemenl des éludes n'est pas rrai des earrières professionnclles, mais il n'est que trop récl dans le domaine de la science elle-même.

On est frappé, quand on parcourt les programmes des universicés allemandes de l'extrème multiplicité de l'enseignement dans toutes les branchej des connaissances humaines et surtout dans les facultés des seiences el de philosophie, lesruelles constituent en définitive le fondement de toutes les sciences humaines. Ainsi tous les talents, toutes les aptitudes des professeurs trouvent à se produire: ainsi des idées neuves, des ćcoles nowrelles pewrent s'établir; ainsi le progrès peut se contimucr dans le scin de ce qui est le plus progressible en ec monde, la 
science, les eonnaissances humaines. Mimsi la jeunesse avide de s'iustruire trouve-t-clle de nombreuses sourecs pour s'abreuver; tous les germes qu'elle apporte arec clle seront fecondés; toutes les aspirations seront soutenues.

Le deroir du gouvernement nous parait étre de multiplier les sotreces de l'enseignement; de faroriser, d'exiger peut-être des cours privés et publics; d'encourager largement les cours libres, d'ourrir des chaires i tous ceux qui sont dignes d'y entrer. Divers moyens ont ćté proposćs et essayés. Le nôtre pourrait être aussi mis à l'épreuve. Il consiste, répétons-le, à fixer à l'université les jeunes talents par la création d'enplois sceondaires arec faculté d'ourrip des comrs anmexes de ceux dont la connaissance est nécessaire pour les cxamens professionnels.

Aussi, en Allemagne encore, il n'est pas de professcur d'une chaire seientifique qui n'ait au moins un assistant. On ne comprendrait même pas comment chez nous on peut s'en passer. Nous avons, il est vrai, la meilleure de toutes les raisons, la nécessité. Les professeurs les plus importants ont mèue deux ou trois assistants.

Par une mesure récente, l'arrèté du 50 janvier 1864 , tous les doctenrs sont autorisćs sous certaines conditions à ouyrir dans les universités de l'Etat des cours privés portant sur des matic̀res complénentaires de l'enseignement officiel. Nous applaudissons à celle décision, et surtout it l'esprıt qui l'a inspirće. Elle est peut-c̀tre trop libérale, en ce sens que nous aurions préférć roir les facultés prendre les garanties dont elles doirent entourer leur autorisation, sous la forme des épreuves du doctorat spécial. Mais nous l'aeceptons telle qu'elle est, dans toute sa libéralité. Reste à saroir quels effets elle produira dans la pratique et quel accueil lui est réservé de la part de ceux qu'elle concerne : les docteurs d'une part, les nniversités de l'autre: les premiers se présenteront-ils: les sceondes autoriseront-elles? Quel esprit animera les uns et les autres?

Mais en supposant que des docteurs soient autorisćs et qu'ils ouvent des cours, quel sort leur est-il réservé? Quel arenir les altend-t-il? De quoi virrout ees jeunes gens et quelle sera leur position? On nous répondra qu'il n’incombe pas au gouvernement de pourvoir aux besoins de tous ceux qui se présentent à lui, el qu'ils doirent trouver le salaire légitime de leur travail académique dans l'inscription qu'ils sont en droit de réclanner de leurs auditenrs; que si leur cours est bon, répond ¿i un besoin récl, il sera suivi et dès lors salarié : que d'ailleurs ils se trouvent dans le droit commun et sont déjà favorisés par l'hospitalité qu'ils recoirent de l'université. Sans doute, tout cela est riti. Mais telle n'était pas notre question. Nous ajouterons cucore que le gourerneunent ne connaissant pas la valeur des candidats qui se présentent pour ouvrir un eours ne saurait se les allacher de suite el que eclle épreure est précisément instituée en vue de permettre aux talents et anx aptitudes de se produire. C'est un arrégariat que l'on eréc et qui se recrule 
non par faveur ou nomination du pouroir, mais librement par me sorte de concours permanent, ce qui vaut bica mieux qu'un concours IIIomentané et aceidentel.

Notre question sera désormais mieux comprise. Que peut espérer le jeune homme de talent, qui aura réussi dans son enseignement prépilraloire? La mort la plus prompte possible de l'un des titulaires du professorat. Cette espérance est malséante, inavouable et sa réalisation souvent à longue éehéanec; elle serait parfois trop ambiticuse. Mais en admettant qu'clle puisse ètre nourrie, de quoi peut vive, pour tout dire en un mot, celui qui se prépare ainsi au professorat. Du produit d'un cours que les étudiants ne doivent pas suivre pour leur cxamen. Cela ne saurait être séricux. Mais ce qui nous parait vrai, e'est que si le jeune homme a du talent, la pratíjue de la médecine, l'exereiee du barreau, les besoins de l'industric ou quelque autre chose l'auront bientôl attiré et distrait de l'université. Si l'on pouvait le fixer par quelque fonction utile d'ailleurs, il ferait dès lors partie de I'Université? Je roudrais qu'on pût le nommer préparateur, conservateur, assistant, ou quelque autre chose semblable. Dès lors il serait rassuré pour son avenir. Non pas qu'il doive néecssairement arriver au professorat dans l'unirersité à laquelle il est attaché, mais continuant it faire preuve de talent, lorsque quelque part dans le pays une position plus ou moins supérieure à la sienne deviendrait vacante, l'opinion publique se porterait sur lui. Pour la botanique les besoins sont grands et les positions nombreuses. Que chacun dans la limite de sa compétence cite les carrières auxquelles telle ou telle science peut conduire.

Il n'en est pas autrement en Allemagne. Les universités se font unc véritable concurrenee et cherehent à s'attirer les savants en renom. La plupart des professcurs passent plusicurs fois dans leur vic d'une université à une autre. Qui agrégé ici, devient professeur extraordinaire ailleurs, puis professeur ordinaire dans une troisième université et à chaque déplacement il voit sa position s’améliorer.

Les agrégés ne jouissent en Allemagne d'aucun traitement et n'interviennent en aucune facon dans l'administration de l'université. Jls n'ont pas ofliciellement les prérogatives du professorat. Cependant ils sont nombreux et donnent des cours importants et fort suivis.

Chez nous, il faut bien le reconnaître, l'esprit scientifique n'est pas aussi vivace qu'en Allemagne. Et puis nous aimons, dans notre esprit pratique, une position stable, quelque mince quelle soit; nous aimons, en outre, à reconnaitre l'intervention d'un pouroir, l'esprit d'initiative n'est pas aussi léveloppé qu'il pourrait l'être. Ici se fait sentir la part d'influence qu'cxerce sur notre esprit notre voisinage méridional.

Jamais, à aucune époque de leur histoire, les universilés ne furent ronsidérées comme faisant parlic de l'administration. Leur aulonomic cst considéréce comme la pratique du grand prineipe de la liberté de la 
seience. En Allemagne ce caractère s'est conservé à peu près dans son intégrité. En France, l'administration nous semble prévaloir trop dans le régime des Aeadémies ou des Facultés, comme on nomme les différents corps qui composent l'Université de France. Quant à nons, nous nous frourons entre l'Allemagne et la Franee et l'inlluenee de ces deux puissants roisins se fait ígalement sentir. Cependant, l'état de la législation est libéral et le Gouvernement, loin de combattre l'esprit d'initiative dans les Unirersités, clicrelic au contraire à le développer. Chez nous, comme en Allemagne, le Gouvernement s'est réserré l'approbation du programme des cours afin de s'assurer si toutes les malières nécessaires pour les examens y sont convenablement professées. Il doit être entièrement satisfiit sous ec rapport, ear les litres des cours sont presque sans exceptions copiés sur les termes mêmes de la loi. Mais il est peut-ètre le premier à regretter de ne voir rien figurer al programme que ce qui doit s'y trouver. Il engage a ouvrir des cours privés et des cours publies, mais son appel est peu entendu. Claque cours est comme un traité général el eneyelopédique de la matière, point de cours spéciaux, de monographies; on ne sort pas du nécessaire professionncl. Sans méconnaitre plusicur's enuses de cet élat de choses, il nous parait que la principale est le trop petit nombre de professeurs; la plupart se trouvent déji trop surchargés par l'accomplissement de leurs fonetions obligatoires. Il faut done chereher à en augmenter le nombre. Bientòt alors viendra l'émulation, l'esprit scientifique sera surexcité; on sortira un peu du cadre officiel et professionnel. Et puis enfin l'antonomic universitaire sera reuforećc. 
NOTES.

\section{L'ENSEIGNEMENT ROTANIQUE A PARIS EN 186!(1).}

Mox Chen Moxsieun Monhen,

...A part le cour's de M. Chatin, ì l'école de Pharmacie, la botanique ne se donne qu'an second semestre qui commenee suivant les écoles, ì des époques différentes. Généralement fin de mai, commenecment d’arril.

La botanique possc̀de à Paris de nombreuses chaires, et trois jardins botaniques.

Les cours sont absoluments libres et gratuils. Je les suis aree une simple carte d'étudiant bénévole qui ne m'a pas coùté un liard. Et encore n'ai-je jamais eu besoin de m'en servir. Des lommes de tout àge, des lommes du monde, des dames, là où l'entrée leur est permise, forment le public de ces cours, public assez souvent variable selon les eaprices de la mode ou du goùt. Généralement un groupe d'auditeurs sérienx assiste avec r'égularité. Le reste est le premier venu, qui reut passer une heure agréable et instructive. Nulle condition u'est requise ì proprement parler, même à l'école de médecine, où pourtant ce sont les élères qui forment la majorité; les autres sont quelques médecins, pharmaciens, herboristes, el quelques liommes de loisir, nombreux à Paris qui suirent par goût les cours et les herborisations.

Sous le titre d'IIistoire naturelle médicale, Mr Baillon donne à l'école de médecine un cours de botanique les lundi, mereredi et rendredi de 11 heures ì midi, depuis la fin de mars jusques au milicu d'août, époque où l'école se ferme. Les étudiants en médecine le suirent en grand nom-

(1) Un de nos amis, jeune et savant doeteur en seienees naturelles, a bien voulu, sur notre demande, pendant qu'il est à Paris, pour eompléter ses ètudes, nous adresser une lettre pour nous renseigner sur l'enseignement de la botanique dans la eapitale de la Franee. Nous eroyons qu'il est utile et intéressant de la publicr ici. 
bre, de 550 à 400 auditeurs approximatirement, avec les curieux, les oisifs, dont j’ai déjì parlé. Son cours se complète par des lierborisations assez fréquentes.

Dimanche dernier 28 juin, nous sommes allés explorer la belle forit de Fontainebleau. Au départ nous étions environ 120. Mais à peine 80 d'entre nous ont suivis jusqu'au bout, entre autres une demoiselle fort couragcuse, ma foi, car il n'y a pas que les étudiants, qui fréquentent les herborisations; un grand nombre d'étrangers y assiste comme aux cours.

Je me suis vainement adressé au secrétariat de l'École pour avoir quelques renseignements sur le nombre des élèves. On n'en fait pas de rapport, ma-t-on dit, puisqu'il n'y a pas d'appariteur pour demander les eartes et pour noter les présences. Et tel cours a 600 auditemrs (le cours de II. Trousscau, par exemple; le grand amphithéitre est alors tout plein), tandis que tel autre, comme celui d'IIygiène, conpte à peine 100 auditeurs. Le cours de $\mathbf{M}$. Baillon est bien suivi; il a, d'après mon ćraluation, de 5 à 400 auditeurs. Il en est de même pour les cours scientifiques de la Sorbonne, pour lesquels les candidats à la licence et au doctorat en sciences, doivent seuls se faire inserire; encore ne les suiventils que quand ils le veulent bien. Cette catégoric d'auditeurs est peu nombreuse : au secrétariat on m'a dit qu'il n'y arait que 6 élèves inserits pour la licence; le reste est formé de curicux, d'auditcurs libres, de vicillards, d'abbés. En somne, un auditoire très-varié, où se trouvent fort peu d'étudiants, fréquente le cours de M. Duchartre, Ies mereredi et les vendredi à midi, du 15 mars au 28 juin. J'ai régulièrement suivi ce cours : de 30 à 80 personnes y assistent. M. Duchartre ne fait pas d'herborisation. Cette annéc il avait pris pour objet du cours : la physiologie végétale. L'annéc prochaine ce sera le tour des familles naturelles et de la gréographic botanique.

M. Chatin professe pendant toute l'annéc, 5 fois par semaine, un cour's de botanique à l'École de Pharmacie. Là il y a moins d'étrangers : Ies élèves sont en plus grande majorité qu'ì l’École de Médecine, puisque l'École de Pharmacic est plus loin du centre. J'ai assisté une fois à ec cours, qui compte r'égulic̀rement de 60 à 80 auditeurs, m'a-t-on dit. II. Chatin traite aussi très-fréquemment de la botanique rurale. J'ai assisté à une herborisation de ec professeur au Bois de Boulogne: nous étions très-nombreux. Un jardin de pharmacic bien entretenu est atteuant à l'école.

Au Muséum d'histoire naturelle, les cours sont absolument lihres, et suivis par des lommes de loisirs et d'études et par des auditeurs d'aventule.

M. Brongniart, sous le titre de Physiologie comparée, y a donné cette annće, depuis le mois de mars, un cour's sur les dicolylédons gymnospermes, les monocotylédons, les cryptogames et les pluntes fossiles, les 
lundi, mereredi et vendredi it 91/2 heures. C'est un cours très-intéressaut, que lat clinique des hòpitaux m'a malheureusement cmpèché de suivre. J'y ai compté de 40 à 30 audileur's.

11. Decaisue est professeur de culture. Il parte des applications de la physiologie végétale à la culture el de l'histoire des végétaux cultivés, les mirrdi, jeudi et samedi à $81 / 2$, depuis le mois de mai. Il a 53 i 30 auditcurs.

M. Georges Ville enseigne la physique végétule au Mtuséum. II traite des conditions et des agents qui déterminent, favorisent et règglent la production des végétaux. Son conr's est considéré comme une préparialion à I'étude pratique des principaux systèmes de culture. Une trentaine d'auditcurs suit son conrs.

Le collége de lrance ne compte pas de professenrs de botanique.

Le conservatoire des Arts eb Métiers est la Sorbonne de la classe ouvrière. Les cours s'y donnent le soir.

Il est inutile de dire qu'ils sont gratuits, libres de toute inseription, de lout contrôle. Pour tout appariteur, un garde de Paris, en faction, y maintient l'ordre. Ils sont très-bien suivis par des ouvrier's et des patrons, el par beaucoup d'autres personnes qui veulent s'instruire.

M. Doussingault y donne la Chimic agricole (action de l'engrais et de l'atmosphìre sur la végétation; alimentation des hom nes et des animaux) le dimanche is $105 / 4$ du matin et le jeudi à 7 1/2 licures du soir.

M. Moll y enseigne l'A gricullure (assolement et rotation; direction de l'entreprise agricole) a $71 / 2$ heures du soir Ies mardi el jeudi, el M. Mantgon, les Travaux agricoles el le génie rural (trivaux du sol, récolte, constructions) ì 7 1/2 heures du soir, les mereredi et samedi.

Vous voyez bien, Honsicur, aux litres seuls de ces cours qu'ils ne rentrent plus dans l'enseignement classique de la botanique.

Outre les cour's, nous avous des conférences. Ainsi M. Georges Ville, dı Ifuséum, a douné quelques conférences agricoles à l'inslitul de Vincennes. J'ai à vous mentionner de plus un cours pratique d'arboriculture au jardin de Luxembourg.

Le jarlin botanique du Muséun est bien tenu: Les serres, l'orangerie sont fort belles. Un musće de botanique se troure aussi dans l'établissement.

Le jardin botanique de l'École de médecine est enclavé dans le jandin dı Luxembourg. Il est paure en espèces : une pétition cireule parmi les itudiants ponr qu'il soit rendu convenable.

Votre bien derour,

Chanles lieyrevs.

loric. 2 juillot 1 stio. 


\section{L'RASEIGNEMENT DE LA BOTANIQUE A LONDRLS EN $186 \%$.}

Cous sommes redevable de ces renseignements it notre savant eollegue et excellent.

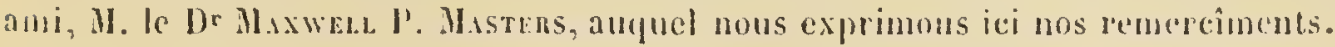

......xford et Cambridge ont des professeurs et des jardins de hotanique. Les élères y sont peu nombreux et les conrs pen étendus. M. Ie I)r Balfour est professcur à Édimbourg et donne en étć une execllente série de lecons, ainsi que des démonstrations pratiques et des excursions. Le jardin botanique y est remarquable et les élèves en grand nombre.

Dublin possède deux jardins botaniques. Celui de l’université se trouve sous la direction du Dr IIarvey. On peut passer sous silenee les petites unirersités d'Écosse et d'Irlande et en venir, de suite, à Londres.

L'université de Londres n'est nullenent un institut d'enseignement, mais elle est sculement chargée des fonctions d'examen. Les grades qu'on y obtient sont fort considérés. Les examinateurs sont des hommes de la plus haute réputation. Les candidats, spécialement pour la médecine, sont très-nombreux. Les examinateurs changent tous les 5 ou 4 ans. Le $\mathrm{D}^{\mathrm{r}}$ Thomson (Flore indice) et le Rev M. J. Berkeley sont actuelIement chargés des examens en botani(jue. Le jury procède par examen écrit et par cxamen oral (viva voce). Les récipiendaires doivent décrire couramment des plantes qu'on leur présente et ils ont à examiner des tissus sous le microscope. L'examen est done essenticllement pratique.

Il n'y a d'autres conrs publies de botanique à Londres que ecux qui sont professés dans les écoles de médecine. Londres possède 10 à 12 grands hòpitaux, à chacun desquels est annexće une école de médecine. Nous n'arons done pas, comme en Belgique, une grande institution scientifiqne d'enseignement, mais plusicurs écoles libres. Chaque hôpital ou chaque collége possède un professeur ou un tecteur en botanique. La plupart donnent pendant l'été un cours d'une cinquantaine de leçons, depuis le mois de mai jusqu’à la fin de juillet. Des démonstrations ou des lierborisations ont lien toutes les semaines ou tous les quinze jours.

Voici quelques renseignements particulicrs.

Mi. le professcur Oliver enscigne avec beaucoup de talent au Collége de l'université, où il a remplacé M. le $\mathrm{D}^{\mathrm{r}}$ Lindley.

II. Ie Dr Bentley est professeur au Kings' College.

II. Ic Dr Harris au St. Burtholomews' Hospital.

M. Jolnson au Guys' Hospital.

M. lc Dr Master', à l'hópital St. Georges.

M. Syme, au Westminster IIospital.

M. le Dr Speneer Cobbald au Middlesex Hospitul.

II. Ic Dr Dresser au St. Mary's IIospital, etc. 
Dans les eolléges de moindre importance la botanique est, en général, professée par de jeunes médecins, désircux de diriger plus tard une clinique.

Le nombre des élèves varie beaucoup suivant les eours. On peut l'évaluer approximativement à une einquantaine par an, dans chacun des quatre principaux hôpitaux qui sont ceux de St. Bartholomé, de Guy, du Roi et de l'Université; en le portant à 50 environ pour chacun des autres on arrive it admettre que 5 ou 4.00 jeunes gens suivent it Londres, année moyenne, les eours de botanique.

L'enseignement pour chaque cours se paie ordinairement trois guinées, mais la plupart des étudiants versent en une fois la somme de 100 lives sterling pour tous les frais de leurs études jusqu'à l'obtention du titre de docteur en médecine et en chirurgie.

Chaque professeur de botanique dispose pour ses élèves de spécimens, de diagrammes, d'herbiers, de microscopes et d'autres moyens d'étude. Il les conduit, en outre, à Kew, au jardin de la Société botanique à Regents I'ark, au jardin des Apothicaires a Chelsea. Ce deruier a pour' jardinier chef M. Thomas Moore. A Kew, les riches herbiers de sir W. Hooker et de M. Bentham sont accessibles au public. Le petit herbier du British Mruseum est également fort usuel.

On voit, en résumé, d'après ce qui précède, qu'il n'y a d'autre enseignement public de la botanique à Londres que celui des hôpitaux, où il est, d'ailleurs, dirigé spécialement, en vue des nécessités de la carrière nédicale. Les écoles du collége universitaire et du collége royal possc̀dent toutefois un ensemble de cours assez complet, théologie, droit, littérature, mathématiques, médecine, de manière qu'clles replésentent assez bien l'équivalent d'une université.

Londres. Ie 28 juillet 1863. 





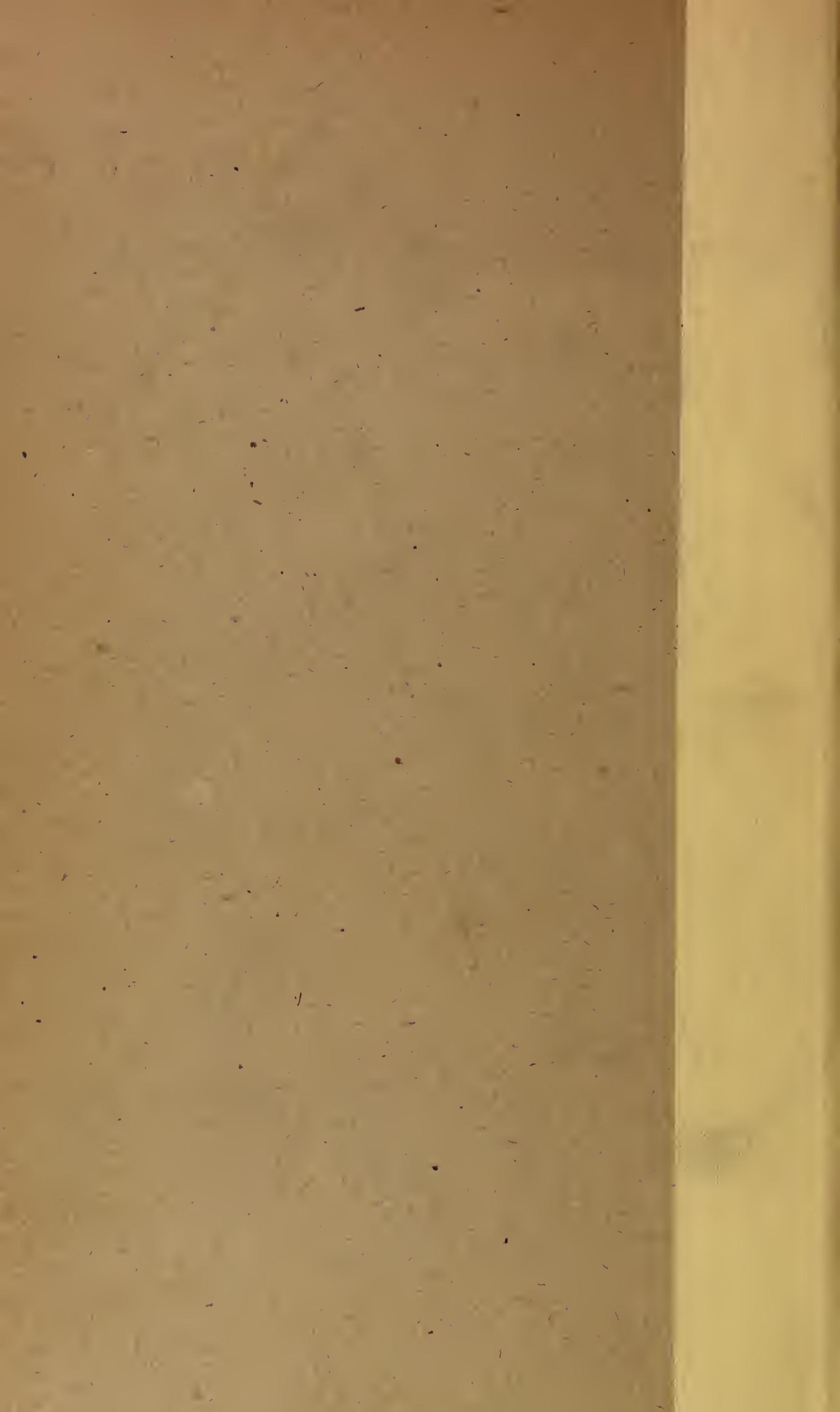

